SPECTROPHOTOMETRY OF 3C 232 AND 3C 249.1

D. Dultzin-Hacyan

Instituto de Astronomía

Universidad Nacional Autónama de México

Apartado 70-264

México, D. F. 04510, MEXICO

ABSTRACT. We find indication of variability of the ratio Mg II 2934/2798 for 3C 232 and discuss the excitation mechanism of Mg II 2934. For 3C 249.1 we identified the following new lines: [Ne V] 3346, [Ne V) 3426 and He I 3884. Comments are made on continuum shapes; in both cases optical continuum variability is confirmed.

\title{
1. OBSERVATIONS
}

The observations were carried out in March 1982 with the $2.1 \mathrm{~m}$ telescope of the OAN, San Pedro Martir, B. C. (MÉxico), using a low dispersion spectrograph coupled with the Optical Multichannel Analyzer described by Firmani and Ruiz (1981).

\section{EMISSION LINE SPECTRA}

\subsection{TON 469 (3C 232)}

TABLE 1

\begin{tabular}{cccc}
\hline Line & $\lambda_{\text {rest }} \stackrel{\circ}{(\AA)}$ & $\begin{array}{c}I_{\text {obs }}\left(x^{\left.-10^{-14}\right)}\right. \\
\left(\operatorname{erg} \mathrm{cm}^{-1}\right)\end{array}$ & $W_{\text {obs }}(\stackrel{\AA}{A})$ \\
\hline MG II & 2798 & $23 \pm 5.0$ & $148 \pm 26$ \\
MG II & 2932 & $13 \pm 3.0$ & $119 \pm 15$ \\
[O II] & 3727 & $5 \pm 0.1$ & $70 \pm 1$ \\
\hline
\end{tabular}

Grandi and Phillips (1978) have suggested two possible fluorescence mechanisms. for the excitation of Mg II $\lambda \lambda 2929,2936$ : Ly $\beta$ an N V Fluorescence. Several observational predictions have not been confirmed for the case of Ly $\beta$ fluorescence: the presence of Mg II $\lambda 1752$ and $\lambda 1737$ 
with a strength comparable to $\lambda 2798$ and also the presence of OI $\lambda 1302$. (See Dultzin-Hacyan, Salas and Daltabuit, 1982).

our observed ratio Mg II 2934/2798 (see Table 1) is $0.56 \pm .20$, a factor of two higher than that observed by Grandi and Phillips (1978). If this ratio is varying with time, simultaneous UV and optical observations are needed to establish whether $\mathrm{N} \mathrm{V}$ fluorescence is responsible for the excitation of Mg II $\lambda 2934$ (Dultzin-Hacyan, 1985).

\section{$2.23 C \quad 249.1$}

TABLE 2

\begin{tabular}{|c|c|c|c|}
\hline Line & $\lambda_{\text {rest }}(\stackrel{\circ}{\AA})$ & $\begin{array}{l}I_{\text {obs }}\left(x 0^{-14}\right) \\
\operatorname{erg} \mathrm{cm}^{-2} \mathrm{~s}^{-1}\end{array}$ & $\mathrm{w}_{\mathrm{abs}}(\stackrel{\circ}{\mathrm{A}})$ \\
\hline [Ne V] & 3346 & $0.9 \pm 0.20$ & $5 \pm 1.0$ \\
\hline [Ne V] & 3426 & $1.1 \pm 0.20$ & $6 \pm 1.0$ \\
\hline$[0 \mathrm{II}]$ & 3727 & $2.7 \pm 0.90$ & $16 \pm 5.0$ \\
\hline [Ne III] & 3869 & $2.2 \pm 0.30$ & $13 \pm 2.0$ \\
\hline $\mathrm{He} I+\mathrm{H}_{8}$ & 3889 & $1.8 \pm 0.40$ & $12 \pm 2.0$ \\
\hline [Ne III] $+\mathrm{H}_{7}$ & 3967 & $1.2 \pm 0.08$ & $8 \pm 0.5$ \\
\hline
\end{tabular}

\section{CONTINUUM}

With these two cases we have an example of locally different continuum energy distribution shapes, in the sense that 3C 232 shows the typical optical flat spectrum and the UV is much steeper, whereas for 3C 249.1 the UV (corrected for galactic reddening) is an extrapolation of the optical continuum (Dultzin-Hacyan, 1985). For both quasars, previous data on variability are confirmed.

\section{REFERENCES}

Dultzin-Hacyan, D., Salas, L. and Daltabuit, E., 1982, Astr. and Ap., $111,43$.

Dullzzin-Hacyan, D., 1985, Rev. Mex. Astron. y. Astrof., 11, in press. Firmani, C. and Ruiz, E., 1981, Recent Advances in Observational Astronomy, eds.: H. L. Johnson and C. Allen (México: UNAMM), p. 25. Grandi, S. A. and Phillips, M. N., 1978, Ap. J., 220, 426. 